\title{
On the Odir iterative method for non-symmetric indefinite linear systems
}

\author{
Anthony T. Chronopoulos ${ }^{1, *, \dagger}$ and David Kincaid ${ }^{2}$ \\ ${ }^{1}$ Division of Computer Science, University of Texas, San Antonio, TX 78249, U.S.A. \\ ${ }^{2}$ Department of Mathematics, University of Texas, Austin, TX 78712, U.S.A.
}

\begin{abstract}
SUMMARY
Several Krylov subspace iterative methods have been proposed for the approximation of the solution of general non-symmetric linear systems. Odir is such a method. Here we study the restarted version of Odir for non-symmetric indefinite linear systems and we prove convergence under certain conditions on the matrix of coefficients. These results hold for all the restarted Krylov methods equivalent to Odir. We also introduce a new truncated Odir method which is proved to converge for a large class of non-symmetric indefinite linear systems. This new method requires one-half of the storage of the standard Odir. Copyright (C) 2001 John Wiley \& Sons, Ltd.
\end{abstract}

KEY WORDS: iterative algorithms; Odir method; solving non-symmetric indefinite linear systems

\section{INTRODUCTION}

In this article, we consider a linear system of equations

$$
A x=f
$$

where $A$ is a non-singular non-symmetric real matrix of dimension $n$. If the symmetric part of the coefficient matrix $A$ is positive definite then it is called definite. We define the minimal polynomial of a nonzero vector $v$ with respect to matrix $A$ as the least degree monic polynomial $q_{k}(\lambda)$ so that $q_{k}(A) v=0$.

Conjugate gradient (CG)-type iterative methods for a non-symmetric linear system obtain approximations to its solution by projecting onto subspaces generated by subsets of the vectors $\left\{r_{0}, A r_{0}, \ldots, A^{i} r_{0}, \ldots\right\}$. Some of these iterative methods minimize (at each iteration) an error

\footnotetext{
${ }^{*}$ Correspondence to: A. T. Chronopoulos, Division of Computer Science, University of Texas, San Antonio, TX 78249,U.S.A.

† E-mail: atc@cs.utsa.edu
}

Contract/grant sponsor: NASA NAG; contract/grant number: 2-1383(1999-2000)

Contract/grant sponsor: State of Texas Higher Education Coordinating Board; contract/grant number: ATP 0036580442-1999

Contract/grant sponsor: State of Texas Higher Education Coordinating Board: contract/grant number: TARP-0036580197-1997; ATP-003658-0520-1999

Copyright (c) 2001 John Wiley \& Sons, Ltd.

Received 3 November 1999

Revised 20 May 2000 
functional over the projected subspaces. This property is called the error minimization property. This property guarantees monotone convergence to the solution (maybe after an infinite number of iterations). Various formulations of Krylov subspace iterative methods have been derived and studied. (See, for example, Reference [1-7] and the references therein.) Here we consider the Odir method [7], which can be applied to general non-symmetric matrices. CG-type methods for non-symmetric linear systems usually are proposed in three forms:

(i) The full orthogonalization method (e.g. FOM/GCG-LS/Odir/GMRES $[1,5,7]$ ), where the size of the projected subspace increases by one at each iteration.

(ii) The restarted methods (e.g. GCG-LS $(m) / \operatorname{GMRES}(m) / \operatorname{Odir}(m)[1,5,7])$, where the method completes $m$ iterations in a cycle and then it restarts with an initial vector equal to $x_{m}$.

(iii) The truncated method (e.g., $\operatorname{MIOM}(k) / \operatorname{orthomin}(k) / \operatorname{truncated-Odir}(k)[3,7,8]$ ) stores and uses only the $k$ most recent direction vectors in approximating the solution.

The full orthogonalization methods possess an error minimization property. However, the restarted and the truncated versions may not possess this property. Therefore, these methods may not converge for certain linear systems. The restarted methods have been proved to converge for a class of non-symmetric linear systems $[9,10]$. However, they may fail to converge for several classes of non-symmetric matrices (even for some skew-symmetric matrices) initial-residual-vector combinations [11]. The truncated versions of these methods have been proven to converge for only symmetric positive definite linear systems.

In this article, we prove that the $\operatorname{Odir}(m)$ method (and all other equivalent Krylov subspace methods) converge, for $m$ fixed, for a class non-symmetric indefinite problems. Also, we prove residual error bounds. We then derive a new generalization of the Odir method called the continued $\operatorname{Odir}(m, k)$ method $(\operatorname{COdir}(m, k))$. The $\operatorname{COdir}(m, k)$ method is equivalent to $\operatorname{Odir}(m)$ method, for $1 \leqslant m$ and $k=0$, and it is a truncated method, for $1 \leqslant m, k$. The $\operatorname{COdir}(m, k)$ method is guaranteed to converge for a larger class of matrices than that for which the $\operatorname{Odir}(m)$ method converges.

Throughout this article the English letters $i, j, \ldots, m, n$ and $s$ will denote integers. The indexes $m, k$ are the maximum number of stored Krylov vectors in the restarted and truncated methods, respectively. The role of $m, k$ for the $\operatorname{COdir}(m, k)$ method will be explained later. In Section 2, we review the $s$-step minimal residual method and present convergence theorems for non-symmetric indefinite matrices. In Section 3, we review the Odir method and prove results on the convergence of the $\operatorname{Odir}(m)$ method. In Section 4 , we present the $\operatorname{COdir}(m, k)$ method and prove convergence results. We summarize our results in Section 5.

\section{RESIDUAL ERROR BOUNDS}

In this section, we obtain some residual error bounds for the $\operatorname{Odir}(m)$ method (with $s \leqslant m$ ) for non-symmetric indefinite matrices under the assumption that $A^{s}$ is definite for some $2 \leqslant s \leqslant m$. In proving the results, we use the $s$-step minimal residual ( $s$-MR) method, which is equivalent (in exact arithmetic) to the $\operatorname{Odir}(s)$ method. (See Reference [9].) In finite arithmetic, the $s$ MR method is not useful because it is not stable. It has been proven that the $s$-MR method and the $\operatorname{Odir}(s)$ method converge for definite matrices, all symmetric and skew-symmetric matrices and for a class of non-symmetric indefinite matrices [9]. 
The solution and residual vectors at the $i$ th iteration of the $s$-MR method are given by

$$
\begin{aligned}
& x_{i+s}=x_{i}+a_{i}^{1} r_{i}+\cdots+a_{i}^{s} A^{s-1} r_{i} \\
& r_{i+s}=r_{i}-a_{i}^{1} A r_{i}-\cdots-a_{i}^{s} A^{s} r_{i}
\end{aligned}
$$

where the scalars $a_{i}^{j}, j=1,2, \ldots, s$ are selected so that $x_{i+s}$ minimizes the error function $\mathbf{E}\left(x_{i+s}\right)=\left\|f-A x_{i+s}\right\|_{2}$ over the affine subspace $L_{i}=\left\{x_{i}+\sum_{j=0}^{s-1} a_{j} A^{j} r_{i}, a_{j} \in \mathbb{R}\right\}$.

The methods $s$-MR and $\operatorname{Odir}(s)$ are equivalent (in exact arithmetic) because they minimize the same error function over the same Krylov subspace. Due to lack of any orthogonality between the direction vectors, the $s$-MR method is not computationally robust to implement. However, it is a useful tool in the theoretical analysis of its mathematically equivalent counterparts.

For the matrix $A$, let $M=\left(A+A^{\mathrm{T}}\right) / 2$ and $N=\left(A-A^{\mathrm{T}}\right) / 2$ be its symmetric and skew symmetric parts. The matrix $M^{2}\left(N^{2}\right)$ is symmetric and non-negative (non-positive) definite [5,9]. Let the eigenvalues of a symmetric matrix $B$ of dimension $n$ be denoted by $\lambda_{n}(B) \leqslant \cdots \leqslant \lambda_{1}(B)$. The following convergence result is of interest for non-symmetric indefinite matrices and was proven in [9]:

Theorem 2.1. Assume that the degree of the minimal polynomial of $r_{0}$ satisfies $2 \leqslant s \leqslant m$. If (a) $d=\lambda_{n}\left(M^{2}\right)+\lambda_{n}\left(N^{2}\right)>0$ or (b) $d=-\left[\lambda_{1}\left(N^{2}\right)+\lambda_{1}\left(M^{2}\right)\right]>0$, then the matrix $A^{2}$ is definite and $s-M R$ and $\operatorname{Odir}(s)$ (with $s \leqslant m$ ) converge to the solution. The residuals satisfy

$$
\left\|r_{i+s}\right\|_{2}^{2} \leqslant c_{1}\left\|r_{i}\right\|_{2}^{2}
$$

where $c_{1}=\left[1-d^{2} / \lambda_{1}\left(A^{2 \mathrm{~T}} A^{2}\right)\right]$.

We prove the following convergence result for the $s$-MR method for normal non-symmetric indefinite matrices. This result is of special interest in classifying the class of matrices for which the $\operatorname{Odir}(s)$ method (with $s \leqslant m$ ) takes many steps before any significant residual norm reduction is observed [11].

Theorem 2.2. Assume that the degree of the minimal polynomial of $r_{0}$ is greater than or equal to 8 and the matrix $A$ is normal indefinite, with eigenvalues $\lambda_{j}=\alpha_{j}+\mathrm{i} \beta_{j}$, where $\mathrm{i}=\sqrt{-1}$ and $j=1,2, \ldots, n$. Define the following regions in the $(\alpha-\beta)$ complex plane:(i)-(ii) in Figure 1 and (iii)-(iv) in Figure 2.

(i) the north and south open cone bounded by $\beta= \pm \alpha$,

(ii) the open cones in the complement of region (i),

(iii) the east and west open cones bounded by $\beta= \pm 0.198913 \alpha$ and the north and south open cones bounded by $\beta= \pm 0.668179 \alpha$,

(iv) the open cones in the complement of region (iii).

Then the following statements hold:

(a) If $2 \leqslant s$ and the eigenvalues of $A$ are confined to either region (i) or (ii) (Figure 1), then $s-M R$ and Odir(s) (with $s \leqslant m$ ) converge. Furthermore, the associated residuals satisfy

$$
\left\|r_{i+s}\right\|_{2}^{2} \leqslant c_{2}\left\|r_{i}\right\|_{2}^{2}
$$




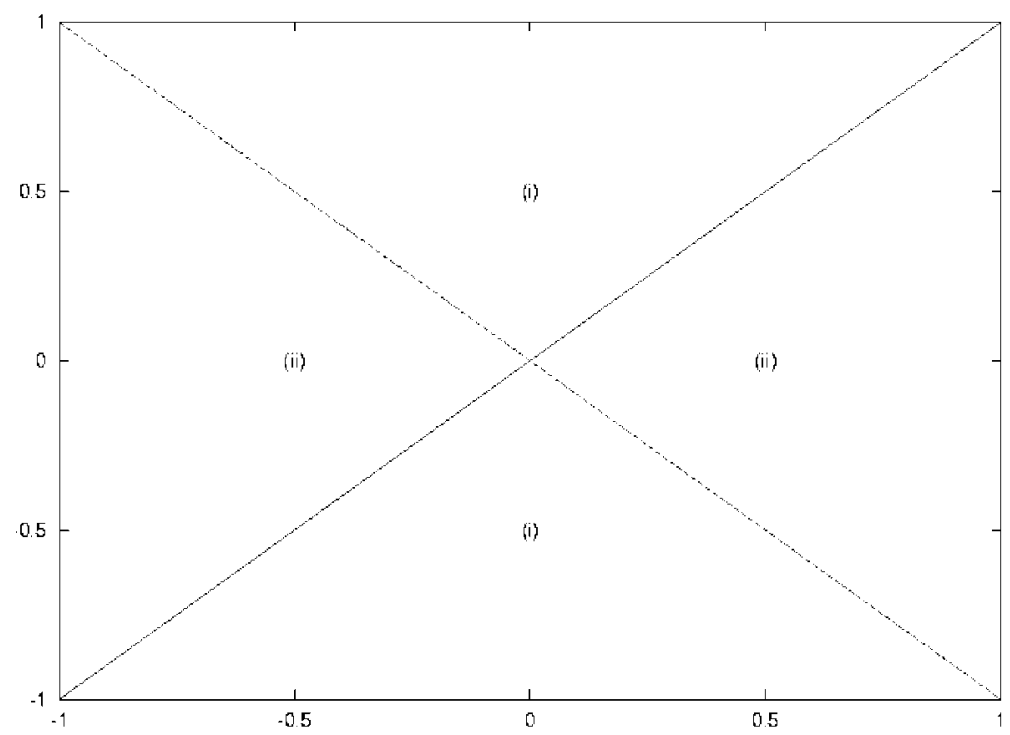

Figure 1. Lines: $\beta= \pm \alpha$.

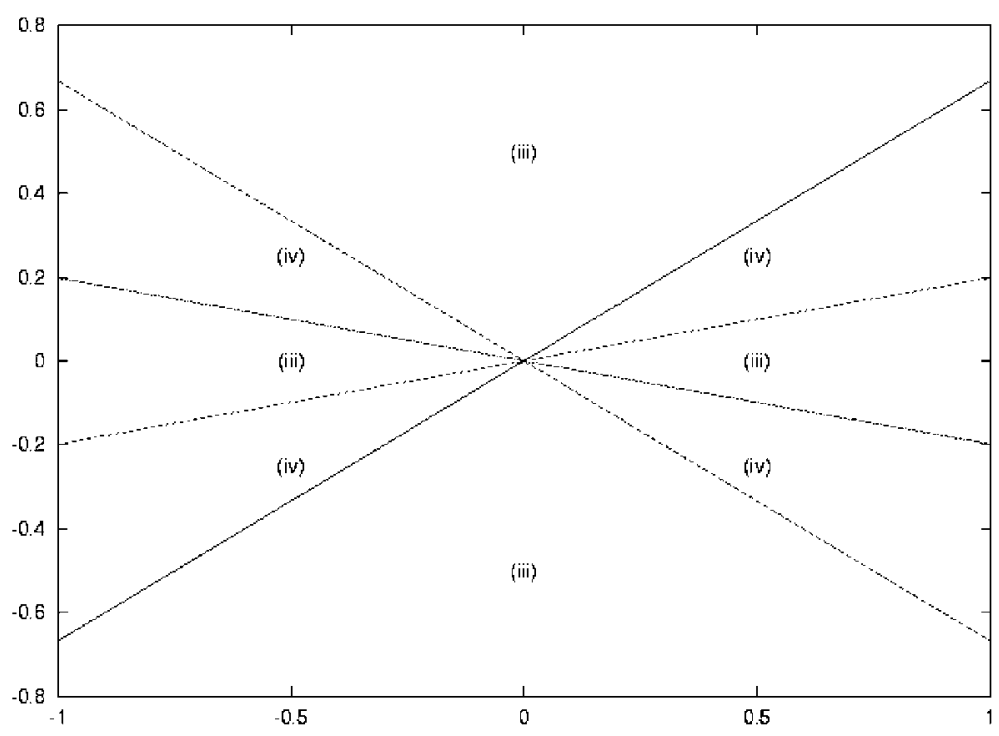

Figure 2. Lines: $\beta= \pm 0.668179 \alpha$ and $\pm 0.198913 \alpha$.

where $c_{2}=\left[1-d^{2} / \lambda_{1}\left(A^{2 \mathrm{~T}} A^{2}\right)\right], d$ is given by

$$
d=\min _{1 \leqslant j \leqslant n}\left\{\left|\alpha_{j}^{2}-\beta_{j}^{2}\right|\right\}>0
$$

(b) If $8 \leqslant s$ and the eigenvalues of $A$ are confined to either region (iii) or (iv) (Figure 2), then the $s-M R$ and $\operatorname{Odir}(s)$ methods (with $s \leqslant m$ ) converge. Furthermore, the associated 
residuals satisfy

$$
\left\|r_{i+s}\right\|_{2}^{2} \leqslant c_{3}\left\|r_{i}\right\|_{2}^{2}
$$

where $c_{3}=\left[1-d^{8} / \lambda_{1}\left(A^{8 \mathrm{~T}} A^{8}\right)\right], d$ is given by

$$
d=\min _{1 \leqslant j \leqslant n}\left\{\left|\alpha_{j}^{8}+\beta_{j}^{8}+70 \alpha_{j}^{4} \beta_{j}^{4}-28\left(\alpha_{j}^{6} \beta_{j}^{2}+\alpha_{j}^{2} \beta_{j}^{6}\right)\right|\right\}>0
$$

Proof

Since $A$ is normal there is a unitary transformation $U$ such that $A=U^{\mathrm{H}} \operatorname{Diag}\left(\lambda_{j}\right) U$. Then

$$
\left(A^{2}+A^{2 \mathrm{~T}}\right) / 2=U^{\mathrm{H}} \operatorname{Diag}\left(\left(\alpha_{j}^{2}-\beta_{j}^{2}\right)\right) U
$$

and

$$
\left(A^{8}+A^{8 \mathrm{~T}}\right) / 2=U^{\mathrm{H}} \operatorname{Diag}\left(\alpha_{j}^{8}+\beta_{j}^{8}+70 \alpha_{j}^{4} \beta_{j}^{4}-28\left(\alpha_{j}^{6} \beta_{j}^{2}+\alpha_{j}^{2} \beta_{j}^{6}\right)\right) U
$$

If we consider only the single steepest descent direction $p_{i}=A^{s-1} r_{i}$ for $s=2,4,6,8$, then

$$
d=\min _{v \neq 0}\left|\left(v, A^{s} v\right) /(v, v)\right|
$$

For the particular choice of $s=2,8$ it is easy to identify the inclusion regions (i) -(iv) and verify that the inequality on the residual norm holds. We prove the case $s \geqslant 8$. For $0 \leqslant l, k$ let $\mu_{l, k}$ be the moments $\left(A^{l} r_{i}, A^{k} r_{i}\right)$. Consider the approximate solution

$$
\bar{x}_{i+1}=x_{i}+\left(\mu_{0,8} / \mu_{8,8}\right) A^{7} r_{i}
$$

then $E\left(x_{i+1}\right) \leqslant E\left(\bar{x}_{i+1}\right)<E\left(x_{i}\right)$ provided that $\mu_{0,8} \neq 0$. Now,

$$
\left|\mu_{0,8}\right|=\left|\left(r_{i}, A^{8} r_{i}\right)\right|=\left|r_{i}^{\mathrm{T}}\left(\frac{A^{8}+A^{8 \mathrm{~T}}}{2}\right) r_{i}\right| \geqslant d
$$

The inclusion region defined by (iii) is determined by solving the inequality

$$
\alpha^{8}+\beta^{8}+70 \alpha^{4} \beta^{4}-28\left(\alpha^{6} \beta^{2}+\alpha^{2} \beta^{6}\right)>0
$$

Equality holds for $\beta= \pm 0.198913 \alpha, \pm 0.668179 \alpha$ (where the slopes are given accurate in six digits).

Note that in Theorem 2.2, we only considered the $s$-MR, with $2 \leqslant s$ or $8 \leqslant s$, and identified the normal matrices for which the method converges. However, the class of normal matrices for which convergence is guaranteed is much larger. This can be shown by verifying that the inclusion regions of $A^{s}$ for $s=4$ or 6 are different from (i)-(iv) and nonempty.

\section{Remark 1}

Let us assume that in a Krylov subspace iterative method

$$
x_{i+s}=x_{i}+a_{i} p_{i}+\cdots+a_{i+s-1} p_{i+s-1}
$$

and the residual $r_{i+s}$ is minimized over the subspace $\left\{p_{i}, \ldots, p_{i+s-1}\right\}$. If the residual can be proved to be orthogonal to $\left\{A r_{i}, \ldots, A^{s} r_{i}\right\}$, then the residual norm bounds of between $\left\|r_{i+1}\right\|_{2}^{2}$ and $\left\|r_{i}\right\|_{2}^{2}$ proved for $s$-MR also apply between the residual norms of $r_{i+s}$ and $r_{i}$ (of the Krylov subspace method under consideration). In this case, we state that the iterations $i, \ldots, i+s-1$ of the iterative method contain one iteration of the $s$-MR method. 


\section{THE ODIR METHOD}

In this section, we review the Odir method and we investigate its convergence properties for non-symmetric indefinite matrices. Odir forms $A^{\mathrm{T}} A$-orthogonal direction vectors and minimizes (at each iteration) the residual norm error $\mathrm{E}\left(x_{i}\right)=\left\|f-A x_{i}\right\|_{2}$ for definite matrices [7]. However, the truncated version of the Odir (TOdir( $k)$ ) method may not converge even if $A$ is definite. For non-symmetric indefinite matrices, the Odir method is guaranteed to converge if $n$ iterations are taken. However, convergence is not warranted for the $\operatorname{Odir}(m)$ method. In this section, we find conditions under which the $\operatorname{Odir}(m)$ method converges for indefinite matrices. Let $x_{0}$ be an initial guess to the solution of (1) and let $r_{0}=b-A x_{0}$ be the initial residual. Let $j_{i}=0$. The Odir algorithm can be summarized as follows.

Algorithm 1. Odir method

compute $r_{0}$; set $p_{0} \leftarrow r_{0}$

for $i=0,1, \ldots$ until convergence do

$$
\begin{aligned}
& a_{i} \leftarrow r_{i}^{\mathrm{T}} A p_{i} /\left(A p_{i}\right)^{\mathrm{T}} A p_{i} \\
& x_{i+1} \leftarrow x_{i}+a_{i} p_{i} \\
& r_{i+1} \leftarrow r_{i}-a_{i} A p_{i} \\
& b_{j}^{i} \leftarrow-\left(A^{2} p_{i}\right)^{\mathrm{T}} A p_{j} /\left(A p_{j}\right)^{\mathrm{T}} A p_{j} \text { for } j_{i} \leqslant j \leqslant i \\
& p_{i+1} \leftarrow A p_{i}+\sum_{j=j_{i}}^{i} b_{j}^{i} p_{j} \\
& A p_{i+1} \leftarrow A^{2} p_{i}+\sum_{j=j_{i}}^{i} b_{j}^{i} A p_{j}
\end{aligned}
$$

endfor

In the Odir method, the storage requirements for the vectors $p_{i}$ and $A p_{i}$ increase up to the dimension $n$ of the coefficient matrix. In the restarted $\operatorname{Odir}(m)$ method after $k * m$ iterations, we restart the algorithm with initial guess $x_{k * m}$ instead of $x_{0}$. The truncated version of the TOdir $(k)$ method has also been proposed in Reference [7]. The algorithm for TOdir(k) is obtained by setting $j_{i}=\max (0, i-k+1)$. If the matrix is symmetric or skew symmetric, it can be easily proven [7] that the Odir(2) method is equivalent to the Odir method. There are examples of non-symmetric definite matrices for which the $\operatorname{TOdir}(k)$ method does not converge.

The following proposition, which was proved in Reference [7] for definite matrices, relates the direction vectors of the Odir and GCR methods [3]. The definiteness of $A$ was used to prove that all step-lengths $a_{i}$ in the Odir method are non-zero. However for indefinite matrices, some step-lengths may be zero.

The following fact was proved in Reference [7].

Proposition 1. Let $0<i$ and assume that in the Odir method the step-lengths $a_{0}, a_{1}, \ldots, a_{i}$ are not zero, then, for $1 \leqslant j \leqslant i+1$, the direction vectors (generated by the Odir method) are

$$
p_{j}=\frac{-1}{a_{j-1}} \bar{p}_{j}
$$

where $\bar{p}_{0}=p_{0}=r_{0}$ and

$$
\bar{p}_{j}=r_{j}+\sum_{l=0}^{j-1} \bar{b}_{l}^{j} \bar{p}_{l}
$$


and

$$
\bar{b}_{l}^{j}=-\frac{\left(A r_{j}, A \bar{p}_{l}\right)}{\left(A \bar{p}_{l}, A \bar{p}_{l}\right)} \quad(0 \leqslant l \leqslant j)
$$

The following remark explains why this result does not hold for the TOdir( $k)$ method.

\section{Remark 2}

For the TOdir $(\mathrm{k})$ method, it is not possible to prove a similar result involving only $k$ direction vectors. To see this, consider $p_{k+1}=A p_{k}+\sum_{l=1}^{m} b_{l}^{m} p_{l}$. Using the assumptions of Proposition 1 with $i=k+1$, we can obtain $p_{k+2}=-a_{k+1}^{-1}\left[r_{k+2}+\sum_{l=0}^{k+1} b_{l}^{k+1} p_{l}\right]$. This expression involves the direction vector $p_{0}$. Therefore, it is not possible (for general non-symmetric matrices) to map the $\operatorname{TOdir}(\mathrm{k})$ direction vectors into the $\operatorname{Orthomin}(\mathrm{k})$ direction vectors $[3,6]$. Also, using this expression for $p_{k+2}$, we can write the step length

$$
a_{k+2}=\left[-\left(r_{k+2}, A r_{k+2}\right)+b_{0}{ }^{k+1}\left(r_{k+2}, A p_{0}\right)\right] /\left[a_{k+1}\left\|A p_{k+2}\right\|_{2}^{2}\right]
$$

because $r_{k+2}$ is orthogonal to $A p_{k+1} \ldots A p_{1}$. However, $\left(r_{k+2}, A p_{0}\right) \neq 0$, in general. Thus, $a_{k+2}$ maybe zero even for $A$ definite.

We next express the direction vectors $p_{i}$ generated by the Odir (or Odir $(m)$ ) method in terms of preceding direction vectors and $A^{l} r_{i}$, where $0 \leqslant l$ and $r_{i}$ is the residual vectors. Now assume that the residual vector $r_{i+s}$ is $A$-orthogonal to all preceding direction vectors. Then we can conclude that $r_{i+s}$ is $A$-orthogonal to $r_{i}, A r_{i}, \ldots, A^{s-1} r_{i}$. We will say that an $s-M R$ iteration is embedded in s consecutive iterations of the $\operatorname{Odir}$ (or $\operatorname{Odir}(m)$ ) method. This allows us to use the residual error bounds of Section 2. We can then establish convergence and obtain error bounds.

\section{Remark 3}

The direction vectors $p_{j}(1 \leqslant j)$ in the Odir method can be expressed in the form

$$
p_{j}=A^{j} r_{0}+\sum_{l=0}^{j-1} \bar{b}_{l}^{j-1} p_{l}
$$

where

$$
\bar{b}_{l}^{j-1}=-\frac{\left(A^{j+1} r_{i}, A p_{l}\right)}{\left(A p_{l}, A p_{l}\right)} \quad(0 \leqslant l \leqslant j-1)
$$

To see this, one uses Algorithm 1 (Steps 4-5) and the $A^{\mathrm{T}} A$-orthogonality of the direction vectors.

The following proposition expresses the direction vectors in terms of powers of $A$ applied to a residual $r_{j}$ (for $1 \leqslant j$ ) and preceding direction vectors.

Proposition 2. Assume $1 \leqslant i$ and that the step-length $a_{i-1}$ (in the Odir method) is not zero. Then for $i \leqslant j$ the direction vectors are given by

$$
p_{j}=-\frac{1}{a_{i-1}} \bar{p}_{j}
$$


where

$$
\bar{p}_{j}=A^{j-i} r_{i}+\sum_{l=0}^{j-1} \bar{b}_{l}^{j-1} p_{l}
$$

and

$$
\bar{b}_{l}^{j}=-\frac{\left(A^{j-i+1} r_{i}, A p_{l}\right)}{\left(A p_{l}, A p_{l}\right)} \quad(0 \leqslant l \leqslant j-1)
$$

Proof

Recall that the direction vector $p_{i}$ is defined by the recursion

$$
p_{i}=A p_{i-1}+\sum_{l=0}^{i-1} b_{l}^{i-1} p_{l}
$$

Since $a_{i-1} \neq 0$, we obtain that

$$
A p_{i-1}=\left[r_{i}-r_{i-1}\right] / a_{i-1}
$$

and $r_{i-1} \in \operatorname{span}\left\{p_{0}, \ldots, p_{i-1}\right\}$. Therefore, $p_{i}=a_{i-1}^{-1} \bar{p}_{i}$ where

$$
\bar{p}_{i}=r_{i}+\sum_{l=0}^{i-1} \bar{b}_{l}^{i-1} p_{l}
$$

The parameters $\bar{b}_{l}^{i-1}=-\left(A r_{i}, A p_{l}\right) /\left(A p_{l}, A p_{l}\right), 0 \leqslant l \leqslant j-1$ are determined by the $A^{\mathrm{T}} A$-orthogonality of $p_{i}$ against $p_{l}$. Now inductively we can form $p_{j}$ for $i<j$.

We next prove that under the assumption that $A^{s}$ is definite for some $s \leqslant m$, then the $\operatorname{Odir}(m)$ method reduces the residual error norm.

Theorem 3.1. Let $s($ for $0<s)$ be an integer and assume that $\left(v, A^{s} v\right) \neq 0$ for all nonzero vectors $v$. For $j=0,1, \ldots$, assume that the degree of the minimal polynomial of $r_{0}$ is greater than $(j+1)$ s then there exists some $k$ (for $1 \leqslant k \leqslant s$ ) such that the step-length $a_{j s+k-1}$ in the Odir method is not zero and $\left\|r_{j s+k-1}\right\|<\left\|r_{j s+k-2}\right\|$.

Proof

We use induction on $j$. For $j=0$, assume that $a_{i}=\left(r_{i}, A p_{i}\right) /\left(A p_{i}, A p_{i}\right)=0$ for all $0 \leqslant i \leqslant s-1$. Since $r_{i}$ is orthogonal to $A p_{l}$ for $l<i$, using Remark 3, we obtain $\left(r_{0}, A^{s} r_{0}\right)=0$, which contradicts the hypothesis. Thus, $a_{k-1} \neq 0$ for some $k<s$. For $1 \leqslant j$, we must prove that there exists index $i$ such that $j s+1 \leqslant i \leqslant(j+1) s$ with $a_{i-1} \neq 0$. From the induction hypothesis, there is a largest positive integer $l$ such that $j(s-1)+1 \leqslant l \leqslant j s$ and $a_{l-1} \neq 0$. Assume that $a_{i}=0$ for $l<i$ and $r_{i}=r_{l}$. This implies that $\left(r_{i}, A p_{i}\right)=0$. Now applying Proposition 2 and using the orthogonality of $r_{i}$ and $A p_{j}$ (for $j<i$ ), we conclude that $\left(r_{l}, A^{i-l} r_{l}\right)=0$. Because of the assumptions this implies that $i \leqslant l+s-1$. This proves that $a_{j s+k-1} \neq 0$ for some $k(1 \leqslant k \leqslant s)$.

We next use this theorem and the results of Section 2 to obtain residual error bounds for the $\operatorname{Odir}(m)$ method. 
Corollary 3.1. Under the assumptions of Theorems 2.1 and 2.2, the following residual error bounds are obtained after iterations of the Odir( $m)$ method (with $m=k s$ ) for one full cycle

$$
\left\|r_{m}\right\|_{2}^{2} \leqslant c_{v}^{k}\left\|r_{0}\right\|_{2}^{2}
$$

where $c_{v}, v=1,2,3$, are the residual norm bounds constants in Theorems 2.1 and 2.2.

Proof

Using Remark 3, Proposition 2, and Theorem 3.1 for $0 \leqslant j \leqslant k-1$, we can show that $r_{(j+1) s}$ is orthogonal to $A^{l} r_{j s}$, for $1 \leqslant l \leqslant s$. This implies that the iterations $j s+l$, for $0 \leqslant l \leqslant s-1$, contain one iteration of the $s$-MR method. Now using repeatedly Theorem 3.1 and the residual error bounds of Theorems 2.1 and 2.2, we obtain the result.

\section{THE CODir $(m, k)$ METHOD}

The $\operatorname{Odir}(m)$ method can be modified to eliminate the need to compute/store the sequence of vectors $\left\{p_{i}\right\}$, for $i \geqslant 0$. (See $[12,13]$.)

Following the methodology in Reference [12], we compute an orthonormal set $V=$ $\left\{v_{1}, v_{2}, \ldots\right\}$, with $v_{1}=A p_{0} /\left\|A p_{0}\right\|_{2}=A r_{0} /\left\|A r_{0}\right\|_{2}$. We set $\rho_{11}=\left\|A r_{0}\right\|_{2}$. We then apply the modified Gram-Schmidt (MGS) method to obtain $v_{j}$, for $1<j \leqslant m$ :

$$
A v_{j-1}=\sum_{i=1}^{j} \rho_{i j} v_{i}
$$

The vectors $v_{j}$ are essentially the vectors $A p_{j}$ of Algorithm 1. Let $A \hat{V}^{m}$ denote the matrix $\left[A r_{0}\right.$, $\left.A v_{1}, \ldots, A v_{m-1}\right]$. Then the following equality holds:

$$
A \hat{V}^{m}=V^{m} R^{m}
$$

where

$$
R^{m}=\left[\begin{array}{ccc}
\rho_{11} & \ldots & \rho_{1 m} \\
& \ddots & \vdots \\
& & \rho_{m m}
\end{array}\right]
$$

and $V^{m}=\left[v_{1}, \ldots, v_{m}\right]$. At the $m$ th iteration, we compute the residual vector as follows:

$$
r_{m}=r_{0}-V^{m} w^{m}
$$

where $w^{m}=\left[\left(r_{0}, v_{1}\right), \ldots,\left(r_{0}, v_{m}\right)\right]^{\mathrm{T}}$.

If we assume that the degree of the minimal polynomial of $r_{0}$ exceeds $m$ then $V^{m}$ is orthonormal and $R^{m}$ is nonsingular. The residual vector $r_{m}$ equals

$$
r_{0}-V^{m} R^{m} y^{m}=r_{0}-A \hat{V}^{m} y^{m}
$$

where $y^{m}=\left(R^{m}\right)^{-1} w^{m}$. Thus, we have $x_{m}=x_{0}+\hat{V}^{m} y^{m}$.

Let us fix $m$. Then the arrays $w^{m}$ and $y^{m}$ are of size $m$ and the arrays $V^{m}$ and $R^{m}$ are of sizes $n \times m$ and $m \times m$, respectively. We plan to use the arrays $V^{m}, R^{m}, w^{m}$ and $y^{m}$, in an outer-inner iteration. Thus, we introduce a subscript denoting the outer iteration index. For example, for outer iteration $l$ we have $V_{l}^{m}, R_{l}^{m}, w_{l}^{m}$ and $y_{l}^{m}$. Also, in order to simplify our notation, we drop the superscript $m$ from these arrays and use $V_{l}, R_{l}, w_{l}$ and $y_{l}$, instead. 
For outer iteration $l=2$, we start from the vector $r_{m}$ and we construct arrays $V_{l}, R_{l}$, in the same fashion as for $l=1$. Let $A \hat{V}_{2}$ denote $\left[A r_{m}, A v_{1}, \ldots, A v_{m-1}\right]$. At outer iteration $l=2$, we apply the MGS method three times:

(a) to orthonormalize $A \hat{V}_{2}$ :

$$
A \hat{V}_{2}=V_{2} R_{2}
$$

(b) to orthogonalize the set $V_{2}$ against the previous set $V_{1}$ :

$$
\tilde{V}_{2}=V_{2}+V_{1} B_{(2,1)}
$$

where $B_{(2,1)}$ is an $m \times m$ array and

(c) to orthonormalize $\tilde{V}_{2}$ in order to obtain the final set $\bar{V}_{2}$ :

$$
\tilde{V}_{2}=\bar{V}_{2} \bar{R}_{2}
$$

We note that if we orthogonalize $V_{2}$ against only $m 1$ vectors in $V_{1}$ (with $m 1<m$ instead of all the $m$ vectors), then the matrix $B_{(2,1)}$ will have $(m-m 1)$ zero rows. For $l=1$, $\bar{R}_{1}=R_{1}$ and $\bar{V}_{1}=V_{1}$.

We are now ready to compute $r_{2 m}$ as follows:

$$
r_{2 m}=r_{m}-\bar{V}_{2} w_{2}
$$

where $w_{2}=\left[\left(r_{m}, \bar{v}_{(2,1)}\right), \ldots,\left(r_{m}, \bar{v}_{(2, m)}\right)\right]^{\mathrm{T}}$. We note that

$$
r_{2 m}=r_{m}-\bar{V}_{2} \bar{R}_{2} \bar{R}_{2}^{-1} w_{2}=r_{m}-\tilde{V}_{2} \bar{R}_{2}^{-1} w_{2}=r_{m}-\left(V_{2}+V_{1} B_{(2,1)}\right) \bar{y}_{(2,2)}
$$

where $\bar{y}_{(2,2)}=\bar{R}_{2}^{-1} w_{2}$.

We can now express $r_{2 m}$ in terms of $A \hat{V}_{1}$ and $A \hat{V}_{2}$ :

$$
r_{2 m}=r_{m}-A \hat{V}_{2} y_{(2,2)}-A \hat{V}_{1} y_{(2,1)}
$$

where $y_{(2,1)}=R_{1}^{-1} B_{(2,1)} \bar{y}_{(2,2)}$ and $y_{(2,2)}=R_{2}^{-1} \bar{y}_{(2,2)}$. Thus, we can obtain $x_{2 m}$ :

$$
x_{2 m}=x_{m}+\hat{V}_{2} y_{(2,2)}+\hat{V}_{1} y_{(2,1)}
$$

In the discussion above, we have presented the first $2 m$ iterations (or the first two outer iterations) of the $\operatorname{COdir}(m, k)$ method, for $k \leqslant m$.

We denote by the $\operatorname{COdir}(m, \infty)$ method the algorithm when no truncation is performed. We will show later that the $\operatorname{COdir}(m, \infty)$ method is equivalent to the Odir method. At outer iteration $l$ of the $\operatorname{COdir}(m, k)$ method, we must store the $k$ vectors of preceding outer iterations. We denote by $l_{k}$ the index of the earliest $V_{j}$ that we orthogonalize the current $V_{l}$ against. We have: $l_{k}=0$ for the $\operatorname{COdir}(m, \infty)$ method and $l_{k}=\max (0, l-(k / m+1)+1)$ for the $\operatorname{COdir}(m, k)$ method.

Algorithm 2. COdir $(m, k)$ method

$$
\begin{aligned}
& i 1 \leftarrow 0 ; i 2 \leftarrow m \\
& \text { compute }\left\|r_{i 1}\right\|_{2} \\
& \text { for } l=1, \ldots \text { until }\left\|r_{i 1}\right\|_{2}<\varepsilon \text { do (Outer-Iteration) } \\
& \quad \text { 1. } A \hat{V}_{l} \equiv\left[A r_{i 1}, A v_{1}, \ldots, A v_{m-1} \leftarrow \leftarrow V_{l} R_{l}\right. \text { (via MGS) } \\
& \quad \text { 2. } \tilde{V}_{l} \leftarrow V_{l}+\sum_{j=l_{l}}^{l-1} V_{j} B_{(l, j)}(\text { via MGS) } \\
& \quad \text { 3. } \tilde{V}_{l} \leftarrow \bar{V}_{l} \bar{R}_{l}(\operatorname{via} \mathrm{MGS}) \\
& \quad \text { 4. } w_{l} \leftarrow\left[\left(r_{i 1}, \bar{v}_{(l, 1)}\right), \ldots,\left(r_{i 1}, \bar{v}_{(m, l)}\right)\right]^{\mathrm{T}}
\end{aligned}
$$




$$
\begin{aligned}
& \text { 5. } r_{i 2} \leftarrow r_{i 1}-\bar{V}_{l} w_{l} \\
& \text { 6. } \bar{y}_{(l, l)} \leftarrow \bar{R}_{l}^{-1} w_{l} \\
& \text { 7. } y_{(l, j)} \leftarrow R_{j}^{-1} B_{(l, j)} \bar{y}_{(l, l)}, \text { for } j=l_{k}, \ldots, l-1 \\
& \text { 8. } x_{i 2} \leftarrow x_{i 1}+\hat{V}_{l} y_{(l, l)}+\sum_{j=l_{k}}^{l-1} \hat{V}_{j} y_{(l, j)} \\
& \text { 9. compute }\left\|r_{i 2}\right\|_{2} \\
& \quad i 1 \leftarrow i 2 ; i 2 \leftarrow i 1+m \\
& \text { endfor }
\end{aligned}
$$

We note that the $\operatorname{COdir}(m, 0)$ method is identical to the $\operatorname{Odir}(m)$ method. We set $k 1=\mathrm{k} / \mathrm{m}$. We note that the parameters $m$ and $k$ are small (e.g. $m<30$ and $k=k 1 \times m$ where $k 1=0$ or 1 or 2). All storage requirements or operations on vectors of size $n$ will be termed vector else they will be termed scalar.

Algorithm 2 requires that we store: $x_{i 1}, \bar{V}_{l}, r_{j}$ and $V_{j}$, for $j=l_{k}, \ldots, l-1$. Thus, in terms of vector storage, Algorithm 2 requires at most $m+k+k 1+1$ vectors to be stored.

The work per outer iteration consists of either scalar work (Steps 6 and 7 ) or vector work (Steps 1-5 and 8): (a) Steps 1-3: $m^{2}(k 1+1)$ dot products and $m^{2} k 1$ vector updates, (b) Steps 4 and 9: $m+1$ dot products, (c) Steps 5, 8, and 9: $m(k 1+1)$ vector updates. Thus, there are $\approx 2 m k+m+k+2$ vector operations per (inner) iteration.

Theorem 4.1. Let us assume that the degree of the minimal polynomial of $r_{0}$ exceeds $m$. Then unless the algorithm has terminated the following are true:

(i) The vectors $\bar{V}_{l}$ are orthogonal to $V_{j}$, for $j=l_{k}, \ldots, l-1$.

(ii) The residual vector $r_{i 2}$ is orthogonal to $\bar{V}_{l}$ and $V_{j}$, for $j=l_{k}, \ldots, l$.

(iii) $x_{i 2}$ minimizes $\left\|r_{i 2}\right\|_{2}$ over the affine subspace $x_{i 1}+\bar{V}_{l}$.

(iv) $\operatorname{Codir}(m, \infty)$ is mathematically equivalent to Odir.

Proof

Property (i) follows from the construction of $\bar{V}_{l}$. Item (ii) follows from (a), the selection of $w_{l},(\mathrm{~b})$, (i), and (c), which is the fact that $r_{i 1}$ is orthogonal to $V_{j}$, for $j=l_{k}-1, \ldots, l-1$. Property (iii) follows from (ii). Item (iv) is proved by induction on the outer iteration. It is obvious for $l=1$. For $1<l$, using the induction and Step 5 of Algorithm 2, we see that $r_{i 2}$ is orthogonal to the Krylov subspace of dimension $l m$.

Corollary 4.1. If the matrix $A$ is symmetric and positive definite, or of the form $I-N$ where $N$ is skew symmetric then the $C O \operatorname{dir}(m, k)$ method is mathematically equivalent to the COdir $(m, 1)$ method and to the truncated-Odir(2) method.

Proof

The proof follows if we show that $B_{(l, j)}=V_{l}^{\mathrm{T}} V_{j}=0$, for $j=l_{k}, \ldots, l-2$, because of the following: (a) $V_{l} \subset\left\{A r_{m(l-1)}, \ldots, A^{m-1} r_{m(l-1)}\right\}$, (b) we can use the fact that $A$ is a special matrix which can be transposed in the dot products $V_{l}^{\mathrm{T}} V_{j}$, and (c) from (ii) of the previous theorem: $r_{m(l-1)}$ is orthogonal to $V_{j}$, for $j=l_{k}, \ldots, l-1$.

Corollary 4.2. Under the assumptions of Theorems 2.1 and 2.2, the COdir(m,k) method with $m=s j$ contains $j$ iterations of an $s-M R$ method. Thus

$$
\left\|r_{(i+1) m}\right\|_{2}^{2} \leqslant c^{j}\left\|r_{i m}\right\|_{2}^{2}
$$

where $c$ is the constant in the error bounds of Inequalities (4)-(6). 
Proof

This result follows from Algorithm 2 and Remark 1.

\section{SUMMARY}

We have proven some convergence results for the restarted Odir method and other equivalent Krylov methods. We then derived a new truncated Odir method, which requires only half of the storage of the standard truncated Odir method. This new method converges for the same class of matrices as does the restarted Odir method.

\section{ACKNOWLEDGEMENTS}

We would like to acknowledge helpful discussions with Professor David Young and his generous advice. He has been a source of inspiration. This research was supported, in part, by research grants from (1) NASA NAG 2-1383 (1999-2000) and (2) State of Texas Higher Education Coordinating Board through the Texas Advanced Research/Advanced Technology Program ATP 003658-0442-1999 at The University of Texas at San Antonio. Also, this work was supported, in part, by grants TARP-003658-0197-1997 and ATP-003658-0526-1999 at The University of Texas at Austin from the State of Texas Higher Education Coordinating Board through the Texas Advanced Research/Advanced Technology Program. Some comments of anonymous referees, which helped improve the presentation of some parts of the paper are gratefully acknowledged.

\section{REFERENCES}

1. Axelsson O. A generalized conjugate gradient, least square method. Numer. Math. 1987; 51:209.

2. Concus P, Golub GH. A Generalized Conjugate Gradient Method for Non-symmetric Systems of Linear Equations, Glowinski R, Lions JL (eds), Lecture Notes in Econ. and Math. systems, vol. 134. Springer: Berlin, 1976; 56.

3. Eisenstat SC, Elman HC, Schultz MH. Variational iterative methods for nonsymmetric systems of linear equations. SIAM Journal on Numerical Analysis 1983; 20:345.

4. Faber V, Manteuffel T. Orthogonal error methods. SIAM Journal on Numerical Analysis 1987; 24(1):170.

5. Saad Y, Schultz M. GMRES: a generalized minimal residual algorithm for solving nonsymmetric linear systems. SIAM Journal on Scientific and Statistical Computing 1986; 7:856.

6. Vinsome P. An iterative method for solving sparse sets of simultaneous equations. Proceedings of Fourth Symposium on Reservoir Simulation, Society of Petroleum Engineers of AIME, SPE 5729, 1976; 149.

7. Young DM, Jea KC. Generalized conjugate gradient acceleration of nonsymmetrizable iterative methods. Linear Algebra with Applications 1980; 34(7):159.

8. Brown PN, Hindmarsh AC. Reduced storage methods in stiff ODE systems. Applied Mathematical Computation 1989; 31:40.

9. Chronopoulos AT, S-step Iterative methods for (Non)symmetric (In)definite linear systems. SIAM Journal on Numerical Analysis 1991; 28(6):1776.

10. Joubert $\mathrm{W}$. On the convergence behavior of the restarted GMRES algorithm for solving nonsymmetric linear systems. Numerical Linear Algebra with Applications 1994; 1(5):427.

11. Greenbaum A, Ptak V, Strakos Z. Any nonincreasing convergence curve is possible for GMRES. SIAM Journal on Matrix Analysis and Applications 1996; 17(3):465.

12. Axelsson O, Makarov M. On a generalized conjugate gradient orthogonal residual method. Numerical Linear Algebra with Applications 1995; 2(5):467.

13. Walker HF, Zhou L. A simpler GMRES. Linear Algebra with Applications 1994; 1(7):571. 